\title{
ANÁLISIS DE LA DEMANDA DEL TURISMO PARA LA GESTIÓN SUSTENTABLE DEL DESTINO EN LAS ISLAS GALÁPAGOS-ECUADOR
}

\author{
Angelica Maria Saeteros* \\ Edson Vicente Da Silva** \\ Veronica Gabriela Calles**
}

\begin{abstract}
La información estadística sobre el turismo es importante para la gestión sustentable del destino y es una herramienta fundamental para la toma de decisiones que permiten generar políticas para planificar destino. El artículo presenta una compilación de estadísticas sobre el perfil del turista que visita Galápagos. El objetivo de esta investigación es analizar la demanda del turismo en Galápagos para la gestión del destino, para lo cual, se realizó un estudio estadístico descriptivo con la totalidad de turistas que llegan a Galápagos, tomados de la encuesta realizada mediante la tarjeta de control de tránsito turístico del Parque Nacional Galápago durante los años 2007 - 2014. En el análisis se observa un crecimiento acelerado del turismo en Galápagos que ha provocado problemas con la sustentabilidad del destino; Ecuador y Estados Unidos encabezan el ranking de visitas, con respecto al modo de transportación del turista se sabe que el turismo nacional prefiere la modalidad de viaje con visitas a los centros poblados y el turismo extranjero a bordo de cruceros con visitas entre islas, la temporada alta es en los meses de junio, julio y agosto y la temporada baja en septiembre; estas características permitieron identificar el perfil del turismo para la gestión del destino por parte de los empresarios y las instituciones rectoras del turismo.
\end{abstract}

Palabras Claves: Destino Turístico, Gestión Sostenible, Perfil del turista, Islas Galápagos.

\section{TOURISM DEMAND ANALYSIS FOR THE SUSTAINABLE DESTINY MANAGEMENT AT GALAPAGOS ISLAND-ECUADOR}

Statistical information on tourism is important for the sustainable management of the destination and its a fundamental tool for making decisions that generate plan a destination policies. This article shows a compilation of touristic statistics about Galapagos Island. The objective of this study was to generate information concerning to tourism trends in Galapagos Islands for destination sustainable management. The statistical research used the universe of tourists arriving to Galapagos Island took from the survey of Galapagos National Park control board tourist transit 2007-2014. In the analysis shows an accelerated growth of tourism in Galapagos has caused problems with the sustainability of the destination; Ecuador and the United States lead the ranking of visits, with respect to the mode of transportation of tourist the national tourist prefers the ground trip and the international tourist prefers cruise trip. The high season is during the months of June, July and August and the low season on September. The sociodemographic characteristics allowed the identification of tourism trends in Galapagos Island. All this information is compiling statically in order to improve the destination management by governing institutions and all the social actors that carry weight the tourism activity.

Keywords: Tourism trends, Galapagos Island, sustainable management, destination, tourism.

\section{ANÁLISE DA DEMANDA TURÍSTICA PARA A GESTÃO SUSTENTÁVEL DO DESTINO ILHS GALÁPAGOS-EQUADOR}

Resumo

A informação estatística sobre o turismo é importante para a gestão sustentável dos destinos turísticos e é uma ferramenta essencial para a tomada de decisão que pode gerar políticas para planejar destino. Este artigo apresenta uma compilação de estatísticas sobre o perfil dos turistas que visitam Galápagos. 0 objetivo da pesquisa foi analisar a demanda do turismo em Galápagos para gerenciamento do destino, para tanto, um estudo estatístico descritivo foi realizado com todos os turistas que chegam em Galápagos, a partir da pesquisa realizada pela placa de controle galápago trânsito turístico Parque Nacional durante os anos de 2007 - 2014. Na análise, verificou-se que o rápido crescimento do turismo em Galápagos tem causado problemas com a sustentabilidade do destino. Equador e os Estados Unidos lideram as visitas do ranking, com respeito ao modo de transporte de turistas sabe-se que o turismo doméstico prefere visitas às cidades enquanto os turistas estrangeiros visitam as lhas a bordo de cruzeiros; a alta temporada é nos meses de junho, julho e agosto e baixa temporada em setembro. Esses recursos ajudaram a identificar o perfil da gestão de destino turístico por empregadores e que regem as instituições de turismo.

Palavras Chave: Destino Turístico. Gestão Sustentável. Perfil do turista. Ilhas Galápagos. Equador.

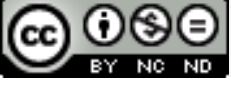

Licenciada por Creative Commons Atribuição Não Comercial / Sem Derivações/ 4.0 / Internacional
* Estudiante del Doctorado en Desarrollo y Medio Ambiente de la Universidad Federal do Ceará. Maestría en Gerencia de Proyectos de Ecoturismo por la Universidad Tecnica de Ambato (2010). Licenciada en Ingenieria en Ecoturismo por la Escuela Superiro Politecnica de Chimborazo (2007). Argentinos 13-20 y Loja, Riobamba / Ecuador. [angelicasaeteros@yahoo.com ]

**Pós-doutor em Planejamento e Geoecologia da Paisagem pela Faculdade de Geografia, Universidade de Havana-Cuba (2007). Pós-doutor em Educação Ambiental pela Faculdade de Educação da Universidade Federal da Bahia (2006). Doutor em Geografia pela Universidade Estadual Paulista Rio Claro São Paulo (1993). Mestre em Planejamento Rural em Função do Meio Ambiente pelo Instituto Agronômico Mediterrâneo de Zaragoza (1987).Graduação em Geografia pela Universidade Estadual do Ceará (1981). Atualmente é professor titular da Universidade Federal do Ceará (1997), tirocínio docente da Universidade Federal da Bahia e professor dos Doutorados e Mestrados em Geografia e de Desenvolvimento e Meio Ambiente (PRODEMA), da Universidade Federal do Ceará. Av da Abolição 2667 ap 707.Meireles - Fortaleza, Ceará / Brasil. [ cacau@ufc.br ]

*** Master en Gerencia de Proyectos de Ecoturismo por la Universidad Tecnica de Ambato (2010). Licenciada en Ingenieria en Ecoturismo por la Escuela Superiro Politecnica de Chimborazo (2007). Docente de la carrera de turismo de la Universidad Autónoma de los Andes. Benjamin Carrión y Victor Emilio Estrada. Riobamba/ Ecuador. [ cristoedygaby@yahoo.com ] 


\section{INTRODUCCIÓN}

El turismo es reconocido como una actividad económica importante, considerada una excelente alternativa para el desarrollo de los territorios (COSTA et al., 2010) que es el ámbito donde se producen los efectos geográficos, sociales, económicos y culturales del turismo (TIMÓN, 2004). Es así que esta actividad ha venido adquiriendo una presencia creciente en la dinámica económica mundial y es considerado un fenómeno en expansión en el mundo donde cada vez es mayor el número de viajeros y el volumen de los recursos económicos que moviliza la actividad (OLIVEIRA et al., 2013). Según la OMT en el año 2015 el mercado turístico marco record de crecimiento en término de llegadas internacionales con un total de 1184 millones de turistas, lo que significa que el turismo sigue siendo la clave para mejorar la economía global, ya que contribuye a generar fuentes de trabajo, el alivio de la pobreza, la protección ambiental, la paz y entendimiento alrededor del mundo. (UNWTO, 2016) y lo que es más para muchos países y regiones del mundo constituye una de sus actividades fundamentales, generadoras de ingreso, empleo y desarrollo. (REBOLLOSO; SALCEDO, 2007).

A pesar de ello el turismo no es un actividad de impacto "cero" sobre el medio ambiente (Peral et al., 2010); por lo que se hace importante analizar el comportamiento de la actividad turística y sus implicaciones, apoyándose en datos históricos que permitan conocer la situación actual, la evolución y el comportamiento futuro de los destinos turísticos (I TOMÁS, 2005) a fin de monitorear los impactos ambientales, sociales, económicos, culturales entre otros e introducir medidas preventivas necesarias para la gestión de los destinos (PÉREZ-LEÓN; CANIVELLCRUZ, 2012). Las estadísticas sobre el turismo no solo se utilizan para la formulación de políticas y estrategias de turismo, sino que las estadísticas oficiales constituyen un elemento indispensable en el sistema de información de una sociedad democrática y proporcionan al gobierno, a la economía y al público datos acerca de la situación económica, demográfica, social y ambiental (LEHOHLA, 2014).

En este contexto, generar información sobre la demanda del turismo en las Islas Galápagos para la gestión sostenible del destino se vuelve necesario, a medida que los impactos antropogénicos sobre el mundo natural siguen aumentando, la importancia de los esfuerzos de conservación para proteger la biodiversidad crece y la conservación es un campo que trasciende los límites tradicionales; produciendo soluciones que abordan las preocupaciones ecológicas, junto con las dimensiones humanas, incluidas las consideraciones políticas, económicas, legales, culturales, estéticas y socio espirituales (CHEUNG, 2015). Además en el ámbito del sistema turístico se nota la actuación del gobierno, de organizaciones no gubernamentales y otras instituciones que conjuntamente componen el espacio institucional y organizacional del turismo (OLIVEIRA ARRUDA et al., 2014) ya que son los entes encargados de la toma de decisiones adecuadas para la gestión y planificación de los destinos (ORGANIZATION, 2004).

El análisis estadístico sobre la demanda del turismo y sus representaciones son una herramienta de planificación que asienten la dinámica del destino y sus adaptaciones (PRAT, 1998). Permitiendo poner más atención a los problemas ambientales y sociales, a la vez buscar nuevas formas y modalidades más participativas, legítimas y eficientes, de "buena gobernanza", asegurando la conservación del destino (BRENNER; SAN GERMAN, 2012).

Las Islas Galápagos son consideradas un laboratorio vivo de biodiversidad y es uno de los destinos turísticos mas visitados del Ecuador. En 1959, un siglo después de que Charles Darwin llego a las islas y sus estudios dieron origen a la teoría sobre la evolución de las especies, el gobierno de Ecuador declaro Parque Nacional al $97 \%$ de las islas y el $3 \%$ para asentamientos humanos y agricultura. En 1978 la Organización de la Naciones Unidad para Educación, Ciencia y Cultura (UNESCO) declaró a las islas Galápagos como uno de los primeros sitios patrimonio mundial de la Naturaleza y en 1985 como Reserva de la Biosfera. En 198670.000 km2 del Océano Pacifico que bordea a las islas fue declarado Reserva Marina de Galápagos y fue expandido en 1998 a 130.000 km2 (DURHAM, 2008)

El turismo en Galápagos inicio a finales de los años 60, en el año de 1968 se abrieron dos vuelos por semana que trasportaba turistas, científicos y colonos; en este mismo año la primera empresa de turismo con modalidad de crucero empezó a ofrecer tours a galápagos, sin imaginar que la inyección de los dólares de los turistas produciría una de las economías de mayor crecimiento en el mundo (EPLER, 2007).

En los años 70 hubo un crecimiento contenido del turismo en el cuál prevalecía el turismo flotante (a bordo de una embarcación) y la infraestructura en tierra era relativamente escasa. Para los años 80 el número promedio de turistas que visitaban Galápagos bordea los 18000. A pesar de que las recomendaciones de los conservacionistas era el de mantener un número mas bajo. Entre los años 80 y 90 la industria turística se posicionó como la fuerza impulsora de la economía de Galápagos desarrollándose rápidamente infraestructura turística que permite hoy por hoy alcanzar cifras que bordean los 225.000 turistas al año (GALÁPAGOS, 2014). 
Este incremento del turismo hace que Islas Galápagos sea un lugar prometedor para la migración que de acuerdo al ultimo censo realizado en el país el 28 de noviembre de 2010, Galápagos tiene una población de 25124 habitantes siendo una de las provincias con mayor crecimiento porcentual de población, particularmente en las décadas de los años 80 y 90 cuando lideraba al resto de provincias con una tasa de crecimiento anual (TCA) que casi alcanzaba el $6 \%$. (LEÓN; SALAZAR, 2011). Para muchos de los residentes actualmente hay una sobrepoblación y muchos casos de migración ilegal a las islas (Muñoz Barriga, 2015) que en su mayoría ocupa puestos de trabajo vinculados directa o indirectamente al turismo. (TAYLOR et al., 2009). El turismo y la migración trae consigo la introducción de especies exóticas a las islas generado impactos negativos en el ambiente que ponen en riesgo la conservación del ecosistema insular (GRENIER, 2012).

En este contexto el Gobierno Ecuatoriano plantea al turismo sustentable, como modelo de desarrollo para Galápagos, el cuál tiene como prioridad la gestión sostenible de los recursos naturales para satisfacer las necesidades socioeconómicas, ambientales y estéticas, respetando la integridad cultural e involucrando a los actores sociales en los procesos de gestión (ARAÚJOSANTANA et al., 2013).

\section{GESTIÓN SOSTENIBLE DEL DESTINO}

La degradación del ambiente, causada por el turismo, se genera a partir de la creciente presión que ejercen los visitantes, sobre los recursos naturales y por la capacidad limitada de estos ecosistemas para recuperarse frente a los impactos recibidos (TISCHER et al., 2015). Para afrontar esta situación, el turismo sostenible, surge, como una opción de desarrollo sostenible que tiene que ver con el aprovechamiento y conservación de los recursos naturales para satisfacer las necesidades de las futuras generaciones y sus necesidades propias (CARDOSO JIMÉNEZ et al., 2014); este concepto fue introducido por primera vez en 1987 por la Comisión Mundial sobre Medio Ambiente y Desarrollo en el informe de Brundtland (BRUNDTLAND, 1987)

En conferencia Mundial de Turismo Sostenible realizada en Lanzarote en 1995, se emite la carta de turismo sostenible en la cuál se contempla que el turismo debe asumir la gestión global de los recursos con el fin de asegurar su durabilidad, permitiendo conservar nuestro capital natural y cultural, incluyendo las áreas protegidas. Siendo el turismo un potente instrumento de desarrollo, puede y debe participar activamente en la estrategia del desarrollo sostenible. Una buena gestión del turismo exige garantizar la sostenibilidad de los recursos de los que depende. (TURISMO SOSTENIBLE, 1995) y que este basado en la idea de que en general la industria debe propender a la sustentabilidad (UNEP, 2005)

El turismo sustentable debe promover que exista un equilibro entre los pilares de la sustentabilidad. Así el desarrollo del turismo sustentable debe ser ecológicamente amigable, económicamente rentable y socialmente equitativo (SCHULTE, 2003); si uno de estos pilares no estaría presente 0 no armonicé el turismo sustentable dejaría de existir en el destino. En este sentido el turismo sustentable se ve como una opción de desarrollo que promueve la conservación de los recursos naturales y se toma como una opción para sustituir las actividades extractivas como la agricultura, ganadería, petróleo, entre otras.

La OMT define al turismo sustentable como aquel que:

[...] "tiene plenamente en cuenta las repercusiones actuales y futuras, económicas, sociales y medioambientales para satisfacer las necesidades de los visitantes, de la industria, del entorno y de las comunidades anfitrionas" (UNWTO, 2004).

Desde esta perspectiva, el ecoturismo o turismo ecológico, como también se le conoce, se vislumbra como la actividad capaz no sólo de coadyuvar a la generación de empleos e ingresos para las poblaciones locales, sino también a la promoción de la conservación y el aprovechamiento del entorno natural y cultural, lo que se traduce en la proliferación de áreas naturales protegidas y en el fomento del ecoturismo como herramienta para lograr el desarrollo sustentable (PINKUS-RENDÓN; PINKUS-RENDÓN, 2015).

La Sociedad Internacional del Ecoturismo inserta al Ecoturismo como un modelo a seguir para alcanzar los objetivos del turismo sustentable y lo define como:

\begin{abstract}
"El viaje responsable a las áreas naturales para conservar el medio ambiente y mejorar el bienestar de las personas locales". Se asume estos conceptos porque el turismo, el medio natural (físico y biológico) y el patrimonio cultural pueden constituir los elementos de una gran fórmula de desarrollo equilibrado para muchas regiones del planeta" (TIES, 1990).
\end{abstract}

El desarrollo sostenible aplicado al turismo trata de llegar a una situación de equilibrio que permita al sector turístico funcionar con un criterio de rentabilidad a largo plazo, pero no a expensas de los recursos naturales, culturales o ecológicos (CARRIÓN LATORRE, 2016). 
En un contexto general de crecimiento de la demanda turística, los ecosistemas se enfrentan al reto de la gestión responsable de las actividades relacionadas con el logro del turismo sostenible, que supone, la gestión propicia del destino, exigiendo una participación activa de todos los agentes implicados en su desarrollo y una mayor dotación de la oferta turística, además del valor de la información turística para su procesamiento objetivo y gobernanza (Sancho PÉREZ; GARCÍA MESANAT, 2011). La gestión sostenible involucra, entre otros factores, el acceso a la información incluyendo las técnicas para buscar, categorizar, modificar y obtener información adecuada y necesaria para la toma de decisiones (ENDERE; ZULAICA, 2015). Contemplando una intersección entre lo público, lo privado y las comunidades locales, siendo el destino la unidad base de gestión para el desarrollo de las políticas turísticas (GONZÁLEZ, 2009).

Para la gestión adecuada de los destinos turísticos es importante analizar el comportamiento de la demanda turística además de considerar la fase de desarrollo. Uno de los modelos mas utilizados es el desarrollado por Butler (1980); quien propuso el modelo de ciclo de vida del destino que se compone de seis fases.

La fase de descubrimiento, es la primera fase de vida, donde el destino es poco conocido solo unos pocos turistas llegan y lo hacen porque huyen de los destinos masificados. Durante esta fase, el entorno natural todavía está sin explotar, así que los accesos a los atractivos turísticos son difíciles (BUTLER, 1980). Los servicios turísticos como hoteles, restaurantes sofisticados, carreteras son escasos. En Galápagos la operación empezó en los 60 s y estaba caracterizada por un buen manejo del parque nacional, investigación científica permanente, guías bien entrenados y una empresa turística justa y bien regulada (HONEY, 2008).

La fase de inicio comienza cuando el flujo de turistas comienza a elevarse, la zona se ha equipado con instalaciones turísticas y comienza a desarrollarse el mercado para los turistas (BUTLER, 1980). Este hecho ocurre en Galápagos a partir de los años 80 s donde la conservación de la vida silvestre en Galápagos empieza a deteriorarse debido al incremento del número de turistas, migrantes y las especies exóticas, la etiqueta de destino ecoturístico desapareció hasta la fecha, considerándose actualmente al turismo como una de las amenazas más importantes para la conservación de la biodiversidad en el archipiélago (MUÑOZ BARRIGA, 2015).

Durante la fase de desarrollo el destino ya cuenta con una llegada de turistas en gran número y durante más tiempo. Ahora el sector turístico se profesionaliza, crece la planta turística y se observa mayor nivel de competencia. La fase de consolidación, es el punto más alto de la vida del destino turístico. La llegada de turistas aumenta pero ya no lo hace de forma exponencial. El destino ya es un clásico en los paquetes organizados por las operadoras del sector y el sector genera altos niveles de venta que permiten una economía a escala (BUTLER, 1980).

La fase de estancamiento aparece cuando se satura elcentro turístico y no evolucionan positivamente ni el número de turistas ni la oferta de alojamiento y puede conducir al definitivo colapso (AGUILAR, 2009). De hecho, en los destinos "maduros" la alta afluencia de turistas empieza a plantear problemas puntuales de saturación turística concentrados espacial y temporalmente (HERNÁNDEZ, 2001) que requieren una planificación y gestión del destino adecuada, prestando atención especial al mantenimiento y conservación de los recursos naturales y artificiales como parte de la oferta (BONILLA; BONILLA, 2007). La fase de post-estancamiento implica la acción de reestructurarse 0 cerrar. La pérdida de visitantes es eminente obligando al destino a recurrir a nuevas estrategias de comercialización. Este modelo puede ser adaptado y analizado considerando las particularidades de cada destino.

\section{METODOLOGÍA}

\section{1 Área de Estudio}

Las Islas Galápagos se encuentran ubicadas en el océano Pacífico a la altura de la línea ecuatorial. El archipiélago tiene su centro geográfico a $0^{\circ} 32.22$ 'S y $90^{\circ} 31.26^{\prime} 0$ (AMADOR, BLIEMSRIEDER, et al., 1996). Las Islas Galápagos fueron reconocidas como provincia en 1993 y están situadas a $1000 \mathrm{Km}$ del Ecuador continental, están conformadas por 234 unidades terrestres emergidas entre islas, islotes con un área total de $8000 \mathrm{Km} 2$, el $97 \%$ de Islas Galápagos es área protegida, cuatro de las islas mayores están actualmente ocupadas por una población de 25124 habitantes (INEN 2010).

Las Islas albergan un alto grado de endemismo tanto de flora como de fauna (BANKS et al 2011.), razón por la que fue declarado Patrimonio Natural de la Humanidad, sin embargo en el 2007 la misma organización colocó a las Islas Galápagos en la lista de patrimonios en peligro de extinción debido a los graves daños que el turismo y la migración han ocasionado, en este mismo año el gobierno ecuatoriano las declaró en estado de emergencia y puso en marcha varios proyectos para analizar el estado del turismo, la migración, especies invasoras y el impacto del desarrollo humano para minimizar el impacto y reducir los peligros sociales y ecológicos de las isla, 
reconociendo la potencial amenaza causada por el turismo (GARCÍA et al., 2013); es así que en junio de 2010 el comité de patrimonio mundial retiró a las Islas Galápagos de la lista de patrimonios mundiales en peligro (UNESCO 2010).

Para garantizar la conservación de la biodiversidad así como el desarrollo sostenible de las poblaciones locales, el gobierno y actores locales consensuaron objetivos comunes, metas y acciones enmarcadas en cuatro componentes principales: gobernanza, reingeniería del destino, mercadeo y monitoreo del turismo promoviendo que la visión a largo plazo del turismo en Galápagos es la transición gradual a un destino de ecoturismo a nivel mundial. Esta visión fue aprobada de manera formal por el Consejo de Gobierno de Galápagos (CGREG) en enero de 2011 al acogerla como política regional para la provincia (GARCÍA et al., 2013).

\subsection{Recolección de Datos}

Este estudio se realizó tomando como base el universo de turistas que llegaron a Galápagos durante los años 2007 - 2014, estos datos fueron tomados de la base de datos de los registros diarios de la Tarjeta de Control de Transito Turístico de Galápagos; estos registros son realizados a todos los turistas que ingresan a Galápagos por vía aérea o terrestre, coordinadamente entre el Parque Nacional Galápagos y el Consejo de Gobierno del Régimen Especial de Galápagos.

\subsection{Instrumentos de recolección de información/ datos}

La tarjeta TCT (Tarjeta de Control de Transito) contiene un cuestionario del perfil general de los turistas y visitantes que viajan a Galápagos (nacionalidad, edad, motivo del viaje, días de permanencia, con quien viaja, tipo de viaje, etc).

\section{Figura 1. Tarjeta de Control de Transito TCT.}

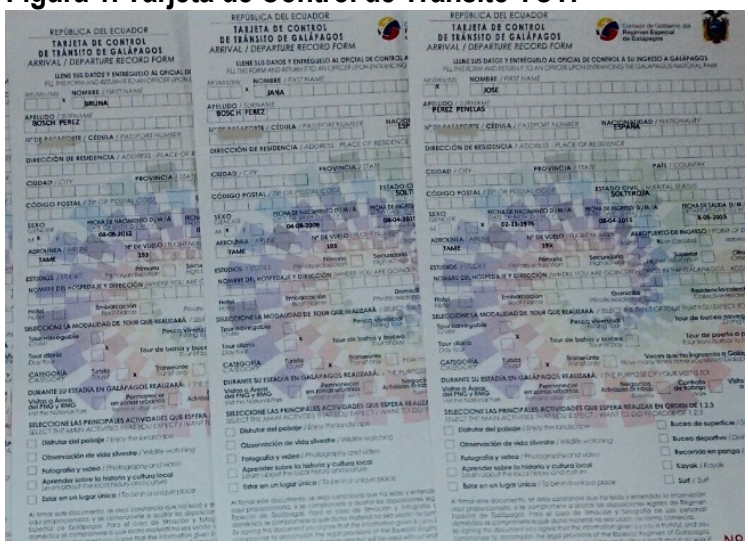

Fuente: Parque Nacional Galápagos y Concejo del Gobierno de Galápagos 2007-2014.
Lo mismo es realizado con una periodicidad diaria, de carácter continuo que recoge información del viaje realizado por el visitante mediante una encuesta directa al turista en el momento de la entrada a las Islas por cualquiera de las vías (aérea o marítima) para después ser entregado en el punto de control de los aeropuertos o puertos.

\subsection{Análisis}

Se realizó un análisis descriptivo de los datos registrados y se identificó las generalidades de la demanda del turismo en Galápagos, para el cuál se utilizó el programa de gestión de bases de datos ACESS 2014 y el software para análisis estadístico TABLEAU 8.1 y EXEL para realizar gráficos.

\section{RESULTADOS - ANÁLISIS DE LA DEMANDA DEL TURISMO EN GALÁPAGOS}

El turismo en Galápagos representa un sector dinámico y en crecimiento (Decasper, 2015) que se refleja en el incremento en el número de turistas que visitaron Galápagos. En el año 2007 se registra la llegada de 161.850 turistas y en el 2014 de 215.691 (Gráfico 1).

Gráfico 1. Número de llegadas de turistas a Galápagos 2007 - 2014.

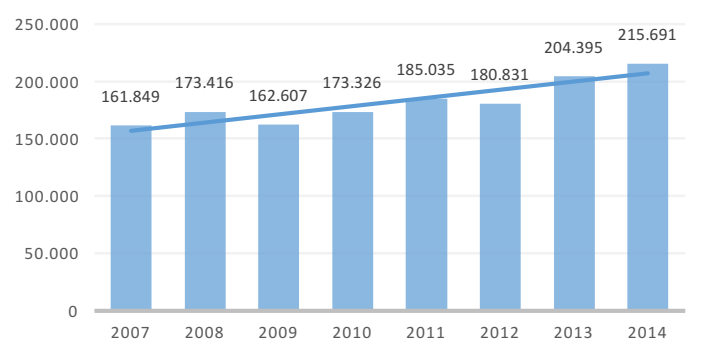

Fuente: Elaboración propia con datos obtenidos de la tarjera de control de tránsito TCT. Parque Nacional Galápagos y Concejo del Gobierno de Galápagos 2007-2014.

La tasa de crecimiento turístico en Galápagos es sostenida en los años 2007 a 2008 y representa el 7.1\%; en el año 2009 al 2010 el $6.6 \%$, en el año 2010 a 2011 con el 6.8\%, en el año 2012 a 2013 con el $13 \%$ que es la tasa más alta registrada y en el año 2013 a 2014 con el $5,3 \%$ con una tendencia marcada de crecimiento en el número de turistas, pero a pesar de este marcado en crecimiento existen años en los que disminuyen las llegadas de turistas como en el 2008 al 2009 con una tasa de crecimiento negativo del $-6,2 \%$ y en año 2011 a 2012 con $-2.3 \%$. La tasa de crecimiento promedio para todo el período 2007-2013 es de 4,7\%. (Gráfico 2). 
Gráfico 2. Tasa de crecimiento de llegadas de turismo a Galápagos.

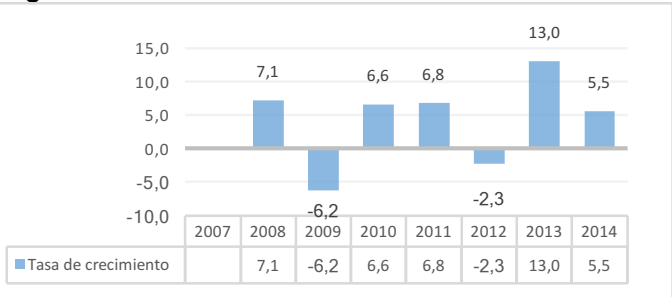

Fuente: Elaboración propia con datos obtenidos de la tarjera de control de tránsito TCT. Parque Nacional Galápagos y Concejo del Gobierno de Galápagos 2007-2014.

A pesar del crecimiento positivo del turismo en Galápagos la economía nacional y global condiciona esta tendencia (BOJANIC e Lo, 2016). Lo que indica que el incremento turístico en la zona de estudio depende de la economía de los países emisores (MARROCU et al., 2015). Los tipos de cambio de moneda son reflejo de las condiciones económicas de un país (LICKORISH; JENKINS, 2010) y el fortalecimiento del dólar ha provocado el aumento de la llegada de turistas procedentes de Estados Unidos como el primer mercado internacional importante en Galápagos generando divisas significativas para el destino y colocando al turismo como un generador económico para el país (CEVALLOS; BURGOS, 2015).

Con respecto al lugar de origen de los turistas que visitan las Islas Galápagos los principales países emisores de turismo provienen de Ecuador $34.34 \%$ y
Estados Unidos $29.27 \%$ los dos suman más del $63 \%$ del total de registro de turistas que llegan a Galápagos. El tercer país en importancia es Reino Unido, aunque representa solamente el $6.20 \%$, seguido de Alemania con el 4.98\%, Canadá con el 4,87\%, Australia 4,21\%. Todos los demás países tienen un porcentaje menor al $4 \%$. Alrededor del $80 \%$ de turistas que visitan Galápagos provienen de un conjunto de 10 países (Top Ten) (Gráfico 3).

Gráfico 3. Origen de llegadas - Top Ten.

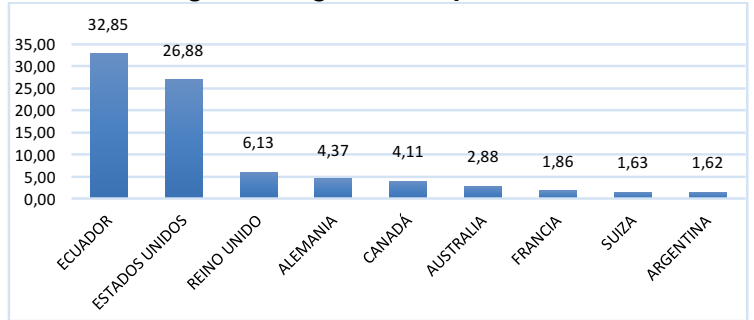

Fuente: Elaboración propia con datos obtenidos de la tarjera de control de tránsito TCT. Parque Nacional Galápagos y Concejo del Gobierno de Galápagos 2007-2014).

A pesar del crecimiento sostenido del mercado norteamericano, en el año 2008, estalla una crisis económica en los Estados Unidos (Nadal, 2008), reflejando una disminución de las llegadas de este mercado emisor que se extiende hasta el año 2010, año en el cuál empieza una recuperación de estos mercados apoyado de las constantes campañas y esfuerzos de promoción del destino.

\section{Gráfico 4. Crecimiento de llegadas de turistas por país.}

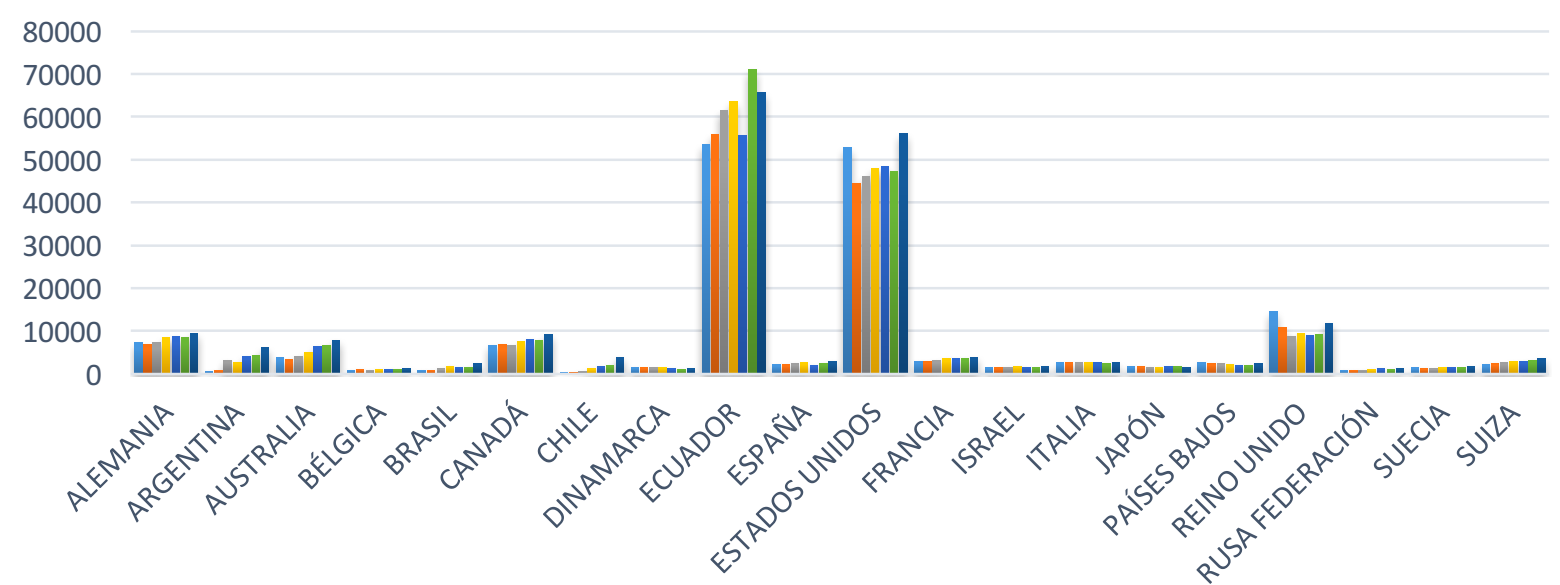

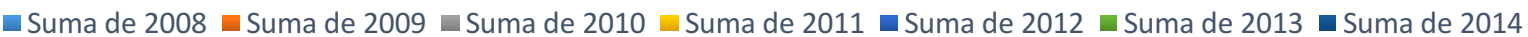

Fuente: Elaboración propia con datos obtenidos de la tarjera de control de tránsito TCT. Parque Nacional Galápagos y Concejo del Gobierno de Galápagos 2007-2014.

Esta crisis global también permitió a Galápagos diversificar su oferta y consolidar algunos mercados emergentes especialmente dentro de la región, como Argentina, Chile y Brasil principalmente, este fenómeno también obedece a la apertura de nuevas rutas y conexiones con países de la región. En el análisis de llegadas de turistas por país se experimenta que la mayoría de países (15 primeros) tienen crecimiento 
general positivo (Gráfico 4). Demostrando que los viajeros internacionales aumentan a una gran velocidad. Las fronteras mundiales parecen ser cada vez un menor impedimento para recorrer el planeta, lo cual genera grandes beneficios tanto para el sector turístico, como para la imagen general del destino (PRIETO, 2014).

El análisis de las llegadas de los turistas reflejan un crecimiento positivo de la demanda y favorecen la diversificación de la oferta de servicios turísticos en el destino, consolidando la economía de familias que se dedican a esta actividad en la localidad, sin embargo la elevada tasa de crecimiento turístico también puede tener repercusiones ambientales y generar stress sobre los ecosistemas y los recursos turísticos naturales de Galápagos, además de los impactos sociales que esto implica, ya que los residentes, intentan sacarle el máximo provecho al turismo al menor costo posible sin importar los impactos causados. Informalidad e ilegalidad constituyen muchas veces la norma.

Este panorama conduce inevitablemente a un decrecimiento en la calidad de los servicios, un incremento descontrolado de la oferta y la consecuente caída de precios y en general el declive del destino al largo plazo, que finalmente implicará menos turistas, más deterioro ambiental y menos beneficios económicos para los residentes (Muñoz Barriga, 2015). Lo que sugiere mayores esfuerzos de planificación municipal e inversión pública para la conservación del destino y al mismo tiempo preguntarse, ¿si en realidad es más conveniente aumentar en número las llegadas de turistas a Galápagos o establecer estrategias para mejorar la capacidad de gasto en el destino y la prolongación de las visitas?

Se debe considerar que la gestión sostenible y adecuada del destino desarrolla estrategias que consoliden la satisfacción de la demanda (Bercial e Timón, 2005). En cuanto a la demanda de turismo internacional el mercado norteamericano es el principal emisor para Galápagos, seguido de Reino Unido, Alemania. El turismo interno supera al internacional (Gráfico 3) por lo que potenciar las llegadas de los propios ciudadanos al destino es importante para el desarrollo sostenible del país. En Galápagos se despliegan estrategias como la puesta en marcha de un nuevo modelo de turismo sostenible en Galápagos, el ecoturismo, que promueve el manejo eficiente del destino y sus recursos turísticos (García et al., 2013) para la gestión turística del destino que permiten potenciar la llegada y permanencia de estos mercados importantes con el análisis continuo de la percepción de los turistas ante los distintos componentes de la oferta, entre los que figuran el mantenimiento y conservación de los atractivos (AMADOR CAYOT, et al., 1996).

Gráfico 5. Tasa de crecimiento por país.

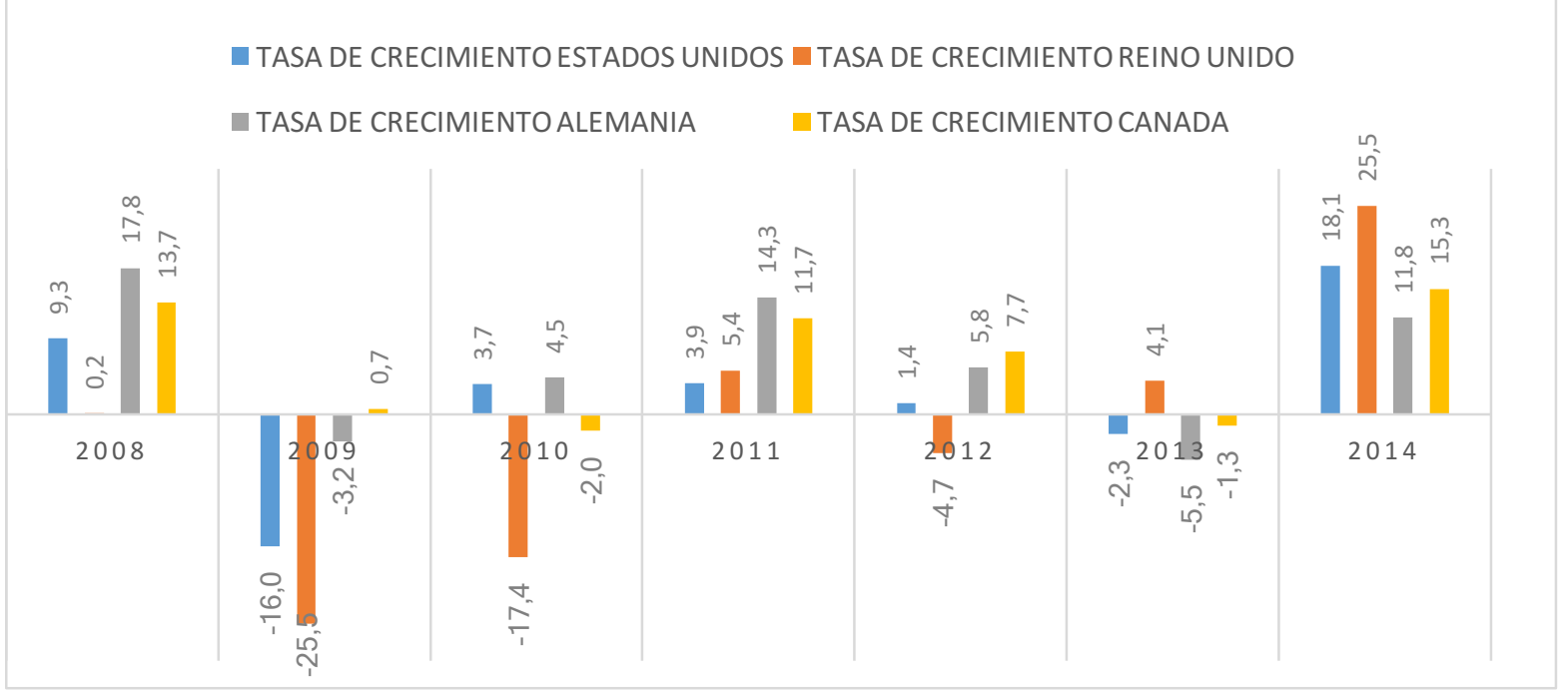

Fuente: Elaboración propia con datos obtenidos de la tarjera de control de tránsito TCT. Parque Nacional Galápagos y Concejo del Gobierno de Galápagos 2007-2014.

En el caso de las corrientes turísticas de los cuatro mercados emisores principales que son Estados Unidos, Reino Unido, Alemania y Canadá, en el año 2009 se experimentó flujos de crecimiento negativo, para el año 2010 se recuperaron dos de los cuatro mercados: Estados Unidos (3.7\%), Alemania (3.7\%); volviendo a caer en el año 2013: Estados Unidos (-2.3\%) y Alemania (-5.5\%). Reino Unido y Canadá tuvieron crecimiento más paulatino con decrecimiento para el Reino Unido en el 2012 (-4.7\%) y en Canadá en el 2013 (-1.3\%). Para el 2014 todos los mercados crecieron: Estados Unidos (10.1\%), Reino Unido 25.5\%, Alemania $(11.8 \%)$ y Canadá (1.3\%) (Gráfico 5).Los países de la región (sur américa) experimenta un elevado de 
crecimiento con tasas promedio de: $56.5 \%$ para Argentina, Chile $45.5 \%$ y Brasil $19.6 \%$, este crecimiento obedece principalmente a dos factores: el primero es la necesidad del destino de consolidar mercados emergentes debido a la crisis global; y el segundo la apertura de nuevas líneas y rutas aérea en la región que facilitan el acceso al destino.

El turismo en Galápagos está caracterizado por dos modalidades de viaje: El turismo "a bordo" basado en pernoctación en un barco $(38,41 \%)$ y el turismo "a tierra" basado en pernoctación en diferentes categorías hoteleras establecidos en los centros poblados $(61,59 \%)$.

Con respecto a la modalidad de viaje y la nacionalidad los datos experimentan una tendencia diferente entre nacionales y extranjeros, los turistas nacionales prefieren mayoritariamente el turismo basado en tierra $(36.36 \%$ ) y a bordo $(1.01 \%)$ lo cuál puede atribuir a la capacidad de pago del turista nacional; mientras que la modalidad de viaje para los turistas extranjeros es a bordo (37.40\%) y en tierra $(25.23 \%)$, ambos no muestran una marcada diferencia sin embargo el porcentaje de quienes realizan turismo a bordo entre nacionales y extranjeros es alta. (Gráfico 6).

\section{Gráfico 6. Modalidad de viaje según procedencia.}

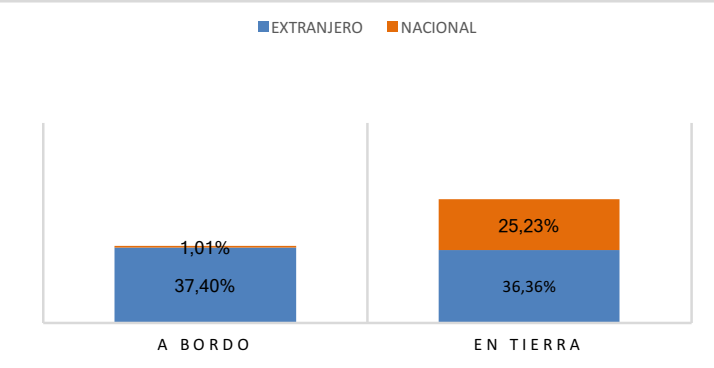

Fuente: Elaboración propia con datos obtenidos de la tarjera de control de tránsito TCT. Parque Nacional Galápagos y Concejo del Gobierno de Galápagos 2007-2014.

La tendencia en la modalidad de viaje ha cambiado durante el periodo 2007 - 2014 mostrando un alto creciendo de el turismo basado en tierra y disminuyendo el turismo a bordo. En el 2007 la proporción de la modalidad del turismo fue $70 \%$ a bordo y $30 \%$ a tierra; mientras que para el 2014 la proporción es 62\% a tierra $38 \%$ a bordo (Gráfico 7 ).

Al analizar la tendencia de la modalidad de viaje se evidencia que el destino turístico Galápagos a evolucionado hacia la perspectiva de un turismo basado en los centros poblados; donde los beneficios económicos que genera esta actividad se redistribuyen en la población; ya que los turistas que realizan sus desplazamientos en tierra consumen los bienes y servicios locales (MANTERO, 2004) y estimulan la economía local generando las condiciones que promueven el desarrollo de las capacidades internas para la producción y progreso de pequeños empresarios (AHMADI et al., 2015).

Gráfico 7. Tendencia de la modalidad de viaje periodo $2007-2014$

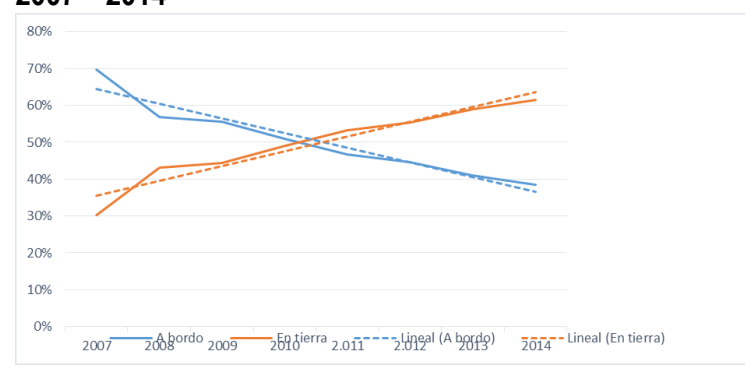

Fuente: Elaboración propia con datos obtenidos de la tarjera de control de tránsito TCT. Parque Nacional Galápagos y Concejo del Gobierno de Galápagos 2007-2014.

Sin embargo, esta es una situación que puede conllevar problemas de planificación así como la perdida de singularidad y exclusividad que caracteriza al destino en Galápagos, ya que las visitas por tierra son menos planificadas y se incrementa la incidencia de oferta de actividades y servicios no regularizados que pueden alterar las condiciones ambientales de los ecosistemas de este destino.

La modalidad de viaje a bordo es más organizada ya que puede ser mejor administrada por los organismos de control que son los encargados del otorgamiento de patentes y cupos de operaciones turísticas así como la planificación de los sitios de visita, sin embargo se ha evidenciado que este tipo de turismo no contribuye a la diversificación de la economía local ya que a pesar de que se pagan patentes de operación los itinerarios y las políticas de esta modalidad no facilitan el consumo de productos y servicios locales.

Los servicios turísticos ofertados en tierra tienen bajos estándares de calidad y apuntan hacía un segmento de mercado constituido por turistas con bajo presupuesto. Por ende, generalmente la oferta directa de la comunidad consigue captar únicamente un porcentaje reducido de los ingresos del turismo. A esto se añade, la presencia de grandes empresas operando cruceros y hoteles de lujo que son las que se llevan la mayor parte de los réditos económicos derivados del turismo y que muchas veces está constituido por capital extranjero. Esta inequidad en el reparto de los beneficios económicos derivados del turismo conduce a una situación de frustración y descontento entre los pobladores (MUÑOZ BARRIGA, 2015).

La duración típica de una estadía es corta: de 4 a 6 días, seguido por estadías de 7 a 9 días y también estadías muy cortas de 1 a 3 días. Comparando la duración de la visita con la modalidad de viaje se conoce que los turistas que escogen la modalidad de turismo basado en tierra tienen una estadía corta por lo tanto 
gastarán menos en el destino, limitando las actividades a realizar y la visita a diferentes centros poblados.

A pesar de que el destino turístico de Galápagos recibe turistas durante todo el año durante el periodo 2007-2014 existe tendencia marcada de una temporada alta en los meses de junio, julio y agosto y una temporada baja en el mes de septiembre, el resto del año se evidencia afluencia heterogénea característica de los destinos turísticos consolidados (Gráfico 8).

\section{Gráfico 8. Estacionalidad.}

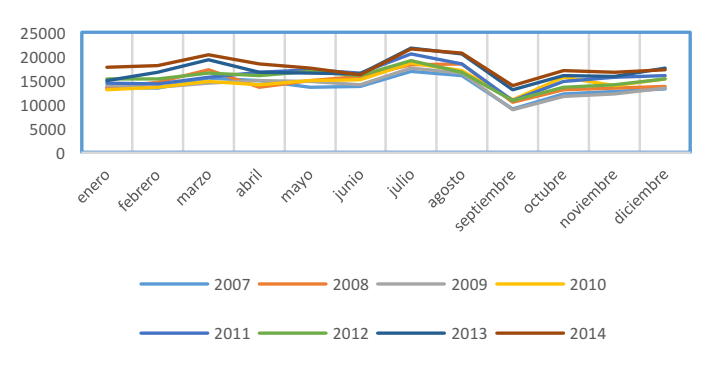

Fuente: Elaboración propia con datos obtenidos de la tarjera de control de tránsito TCT. Parque Nacional Galápagos y Concejo del Gobierno de Galápagos 2007-2014.

A pesar de que las cifras son relativamente positivas, existe la preocupación por mantenerse durante la temporada baja, período en que la demanda disminuye sensiblemente. Ya que el elevado número de turistas durante la temporada alta requiere inversiones de infraestructura imprescindible para el desarrollo de la actividad turística para atender la más diversificada demanda y la falta de uso puede significar un perjuicio general y no sólo económico (GOMES DE MORAES, 2007).

A Galápagos llegan turistas de todas las edades, sin embargo, se evidencia ligeras predominación en llegadas de turistas de 24 a 26 años y de 58 a 70 años, el mercado de adultos mayores está en auge. Estudios aseveran que en las próximas décadas, existirá una disminución en la tasa de natalidad y una esperanza de vida cada vez mayor que dará lugar a un cambio en la estructura de edad dentro de la sociedad. Como tal, los segmentos del mercado de turismo sufrirán cambios tanto de forma cuantitativa y cualitativa. Aunque no se esperan efectos significativos por otros 30 años, los operadores de turismo tienen que empezar a ajustarse a la situación actual y responder de acuerdo con el diseño de sus productos (SCHRÖDER; WIDMANN, 2007).

Además de este grupo de edad existe un elevado grupo de visitante de la edad de 11 años, este efecto se presume que es debido a que en la edad de 11 a 12 años las aerolíneas cambian el valor de las tarifas de niños a adultos (Grafico 9).
Gráfico 9. Edad.

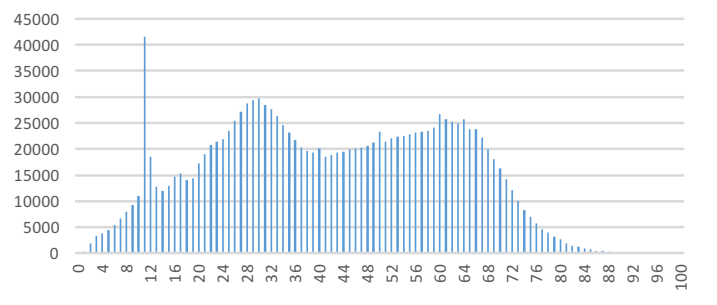

Fuente: Elaboración propia con datos obtenidos de la tarjera de control de tránsito TCT. Parque Nacional Galápagos y Concejo del Gobierno de Galápagos 2007-2014.

\section{CONSIDERACIONES FINALES}

Los resultados obtenidos representan distintas tendencias que obedecen a la dinámica del turismo en las islas Galápagos y su análisis permite generar información y reflexiones para la gestión y planificación sostenible del destino.

Del análisis realizado en este periodo se desprende que el número de turistas que visitan Galápagos ha crecido aceleradamente con una tasa promedio anual del $4.4 \%$. Este incremento tiene implicaciones en la conservación y exclusividad del destino turístico y repercute directamente en el aumento de la población y la dispersión de especies ajenas a las islas colocándola en riesgo para la conservación; esto hace que la planificación y gestión sea más complicada para no ocasionar graves problemas ambientales a los ecosistemas de las islas Galápagos

En referencia al origen de llegadas de turistas a Galápagos, se observa que hay concentración del mercado nacional y en un solo país que es Estados Unidos del mercado internacional, la consolidación de estos mercados obedece a factores externos de la economía global. Conocer las tendencias del mercado en Galápagos permite al destino diversificar su oferta y planificar la consolidación de nuevos mercados que se adapten al modelo de planificación del ecoturismo en Galápagos.

La modalidad de viaje en el periodo analizado ha cambiado totalmente; incrementando la modalidad de viaje de turismo con base en centros poblados (pernoctación en hoteles en las 4 islas) y disminuyendo la modalidad de viaje con base en cruceros a bordo, con esto se presume que generaría mayores beneficios a la población local; sin embargo se evidencia en los datos que las estadías de quienes realizan turismo en tierra son más cortas y son de aquellos países que tienen menor capacidad de gasto, estas visitas se agrupan en lugares y puntos de visita de fácil acceso en los cuales 
se evidencias concentraciones masivas de turistas con repercusiones a la flora y fauna y la exclusividad de la visita; por otro lado la caída notable del turismo a bordo ha ocasionando que grandes inversiones de cruceros se vean amenazadas.

Datos como la edad de los turistas indican tendencias claves para la planificación del destino en función de adaptar las estrategias de satisfacción e interpretación de la visita inclinada a mejorar la conducta de los visitantes y la sostenibilidad en el destino.

La Gestión sostenible del destino implica varios factores que son correspondientes con el modelo de ecoturismo planificado para Galápagos y sus tres ejes que son la conservación del ambiente, el buen vivir de la comunidad (redistribución equitativa de los beneficios económicos generados por el turismo) y la satisfacción del visitante (responsabilidad social), el análisis de los principales indicadores debe incidir en la planificación hacia el logro de la implementación de este modelo en que ninguno de los componentes deben estar por encima de otro sino más bien en armonía.

El estudio de las estadísticas y los indicadores para la gestión de la sustentabilidad de los destinos implica un proceso continuo y participativo, donde actores de diferentes niveles se comprometan en la recolección de datos de calidad a lo largo del tiempo y que deben estar disponible para su uso en todos los niveles especialmente para la investigación.

\section{BIBLIOGRAFÍA}

AGUILAR, C. R. V. El ciclo de vida de um destino turístico: Puerto Vallarta, Jalisco México. CULTUR: Revista de Cultura e Turismo, v. 3, n. 1, p. 1-24, 2009. ISSN 19825838.

AHMADI, M.; ASGARI, S.; GHANAVATI, E. Land capability evaluation for ecotourism development in llam province, a GIS approach. Boletim de Ciências Geodésicas, v. 21, n. 1, p. 107-125, 2015.

AMADOR, E. et al. Plan de manejo del parque nacional Galápagos. Servicio Parque Nacional Galápagos, Instituto Ecuatoriano Forestal y de Áreas Naturales y Vida Silvestre, 1996.

AMADOR, E. et al. Determinación de la capacidad de carga turística en los sitios de visita del Parque Nacional Galápagos. Servicio Parque Nacional Galápagos, Ecuador. 42p, 1996.

ARAÚJO-SANTANA, M. R. et al. Políticas turísticas, actores sociales y ecoturismo en la península de Yucatán. Economía, sociedad y territorio, v. 13, n. 43, p. 641-674, 2013.

BANKS, S. et al. A Review of Galápagos Marine Habitats and Ecological Processes under Climate Change Scenarios. Climate Change Vulnerability Assessment of the Galápagos Islands, 2011 p. 47,
BERCIAL, R. Á.; TIMÓN, D. A. B. Nuevas Tendencias En El Desarrollo de Destinos Turísticos: Marcos Conceptuales y Operativos para su planificación y gestión. Cuadernos de turismo, n. 15, p. 27-44, 2005.

BOJANIC, D. C.; LO, M. A comparison of the moderating effect of tourism reliance on the economic development for islands and other countries. Tourism Management, v. 53, p. 207-214, 2016.

BONILLA, J. M. L.; BONILLA, L. M. L. Diferencias territoriales en la planificación y la gestión del destino turístico. Cuadernos de Turismo, n. 19, 2007.

BRENNER, L.; SAN GERMAN, S. Gobernanza local para el" ecoturismo" en la Reserva de la Biosfera Mariposa Monarca, México. Alteridades, v. 22, n. 44, p. 131-146, 2012.

BRUNDTLAND, G. H. Informe Brundtland. Editorial: OMS Washington, 1987.

BUTLER, R. W. The concept of a tourist area cycle of evolution: implications for management of resources. The Canadian Geographer/Le Géographe canadien, v. 24, n. 1, p. 5-12, 1980.

CARDOSO JIMÉNEZ, C.; CASTILLO NECHAR, M.; HERNÁNDEZ VEGA, C. Sosteniendo al turismo o turismo sostenible (TS): Reflexiones teóricas. Estudios y perspectivas en turismo, v. 23, n. 2, p. 376-395, 2014.

CARRIÓN LATORRE, M. V. Incidencia de la aplicación de prácticas sostenibles en la valoración de la experiencia turística en la Cascada Pailón del Diablo, Parroquia Río Verde, Cantón Baños, Provincia de Tungurahua, 2016.

CEVALLOS, D. M.; BURGOS, R. Z. Alli Shamushka a Ecuador el Turismo Sostenible como Estrategia para el Buen Vivir. Anais Brasileiros de Estudos TurísticosABET, v. 5, n. 2, p. 64-68, 2015.

CHEUNG, $\mathrm{H}$. Ecotourism as a multidisciplinary conservation approach in Africa. Therya, v. 6, n. 1, p. 31-41, 2015.

COSTA, C. C.; OLIVEIRA, I. S. S.; GOMES, L. J. Percepción ambiental como estrategia para el ecoturismo en unidades de conservación. Estudios y perspectivas en turismo, v. 19, n. 6, p. 1105-1120, 2010.

GALÁPAGOS, O. D. T. de. Boletín Observatorio de Turístico. 2014.

DE OLIVEIRA ARRUDA, D.; PASQUOTTO MARIANI, M. A.; MORALES DE QUEIROZ CALEMAN, S. Coordinación y estructuras de gobernanza en un sistema productivo de turismo. Estudios y perspectivas en turismo, v. 23, n. 2, p. 343-361, 2014

TURISMO SOSTENIBLE, C. Conferencia Mundial de Turismo Sostenible. Lanzarote, España, 1995.

DECASPER, S. M. Competitividad y Desarrollo Sostenible en el Turismo. Anais Brasileiros de Estudos TurísticosABET, v. 5, n. 1, p. 47-58, 2015.

DURHAM, W. H. Fishing for solutions: ecotourism and conservation in Galapagos national park. Ecotourism and conservation in the Americas, p. 66-89, 2008.

ENDERE, M. L.; ZULAICA, M. L. Socio-Cultural Sustainability And" Buen Vivir" (Good Living) at Heritage Sites: Assessment Of The Agua Blanca Case, Ecuador. Ambiente \& Sociedade, v. 18, n. 4, p. 265-290, 2015.

EPLER, B. Turismo, economía, crecimiento poblacional y conservación en Galápagos. Elaborado para la 
Fundación Charles Darwin. Recuperado a partir de http://bit. Iy/ONOQWU, 2007.

GARCÍA, J. C.; ORELLANA, D.; ARAUJO, E. El nuevo modelo de turismo: definición e implementación de los principios del ecoturismo en Galápagos. Informe Galápagos 2011-2012, p. 95-99, 2013.

GOMES DE MORAES, A. Análisis de las acciones promocionales de los hoteles para reducir la estacionalidad: El caso de Camboriu-Brasil. Estudios y perspectivas en turismo, v. 16, n. 3, p. 303-322, 2007.

GONZÁLEZ, M. V. Gestión turística del patrimonio cultural: enfoques para un desarrollo sostenible del turismo cultural. Cuadernos de turismo, n. 23, p. 237-254, 2009.

GRENIER, C. Culturas isleñas. Informe Galápagos 20112012, p. 31, 2012.

HERNÁNDEZ, M. G. 124. Capacidad de acogida turística y gestión de flujos de visitantes en conjuntos monumentales: el caso de La Alhambra. Revista PH, n. 36. Especial, 2001.

HONEY, M. Ecotourism and sustainable development: Who owns paradise? Island Press, 2008.

I TOMÁS, P. A. S. Procesos, pautas y tendencias del turismo residencial en las Islas Baleares: ¿ inmigrantes de lujo o turistas de larga estancia? p.281-302. In: Turismo residencial y cambio social: nuevas perspectivas teóricas y empíricas. Universidad de Alicante, 2005.

LEHOHLA, P. Fundamental Principles of Official Statistics: Threats and Responses. High Level Forum on Official Statistics, 2014.

LEÓN, M. G.; SALAZAR, G. C. Población y migración en Galápagos. Informe Galápagos 2011-2012, p. 44, 2011.

LICKORISH, L. J.; JENKINS, C. L. Una introducción al turismo. v. 1ra Edición, p. 286, 2010.

OLIVEIRA, V. M.; GÓMEZ, C. P.; CÂNDIDO, G. A. Indicadadores de sustentabilidad para la actividad turística: Una propuesta de monitoreo usando criterios de análisis. Estudios y perspectivas en turismo, v. 22, n. 2, p. 177-197, 2013.

MANTERO, J. C. Desarrollo local y actividad turística. Aportes y transferencias, v. 8, n. 1, p. 11-38, 2004.

MARROCU, E.; PACl, R.; ZARA, A. Micro-economic determinants of tourist expenditure: $A$ quantile regression approach. Tourism Management, v. 50, p. 13-30, 2015.

MUÑOZ BARRIGA, A. La contradicción del turismo en la conservación y el desarrollo en Galápagos-Ecuador. Estudios y perspectivas en turismo, v. 24, n. 2, p. 399413, 2015.

NADAL, A. La crisis financiera de Estados Unidos. Boletín Económico de ICE, Información Comercial Española, n. 2953, p. 19-29, 2008.

ORGANIZATION, W. T. Indicators of sustainable development for tourism destinations: a guidebook. OWT, 2004.
PERAL, F. J. B. et al. Indicadores sintéticos de turismo sostenible: una aplicación para los destinos turísticos de Andalucia. Rect@: Revista Electrónica de Comunicaciones y Trabajos de ASEPUMA, n. 11, p. 85118, 2010.

PÉREZ-LEÓN, V. E.; CANIVELL-CRUZ, G. The use of sustainability indicators as tools in tourist destination management. 2012.

PINKUS-RENDÓN, M. J.; PINKUS-RENDÓN, M. Á. El ecoturismo: quimera o realidad de desarrollo en la Reserva de la Biosfera Ría Celestún, México. LiminaR, v. 13, n. 1, p. 69-80, 2015.

PRAT, A. G. Nuevas tendencias de ocio y turismo. Papers de turisme, n. 23, p. 88-97, 1998.

PRIETO, L. A. C. Análisis de la gestión de competitividad en Colombia como destino turístico. Revista EAN, n. 76, p. 78-90, 2014.

REBOLLOSO, F. S. M.; SALCEDO, M. P. Turismo, sustentabilidad y certificación: Un reto global. Revista del Centro de Investigación de la Universidad la Salle, v. 7, n. 27, p. 77-91, 2007.

SANCHO PÉREZ, A.; GARCÍA MESANAT, G. ¿Qué indica un indicador? Análisis comparativo en los destinos turísticos. Revista de análisis turístico, n. 2, 2011.

SCHRÖDER, A.; WIDMANN, T. Demographic change and its impact on the travel industry: Oldies-nothing but goldies? In: (Ed.). Trends and issues in global tourism 2007: Springer, 2007. p.3-17.

SCHULTE, S. Guía conceptual y metodológica para el desarrollo y la planificación del sector turismo. United Nations Publications, 2003.

TAYLOR, J. E.; HARDNER, J.; STEWART, M. Ecotourism and economic growth in the Galapagos: an island economywide analysis. Environment and Development Economics, v. 14, n. 02, p. 139-162, 2009.

TIES. TIES \& Ecotourism. http://www.ecotourism.org/tiesecotourism-espa\%C3\%B1ol, 1990.

TIMÓN, D. A. B. El concepto de destino turístico. Una aproximación geográfico-territorial. Estudios turísticos, n. 160, p. 45-68, 2004.

TISCHER, V.; ESPINOZA, H. D. C. F.; MARENZI, R. C. Indicadores socioambientales aplicados en la gestión de ambientes costeros. Caso de estudio Santa Catarina, Brasil. Investigaciones Geográficas, Boletín del Instituto de Geografía, v. 2015, n. 86, p. 53-66, 2015.

UNEP, W. Making tourism more sustainable. A guide for policy makers. Paris and Madrid, UNEP and WTO, v. 8, 2005.

UNWTO. Sustainable Development of Tourism. http://sdt.unwto.org/es/content/definicion, http://sdt.unwto.org/es/content/definicion, 2004.

UNWTO, W. T. O. Annual Report. UNWTO, Madrid, 2016.

\footnotetext{
Processo Editorial / Editorial Process

Editor Chefe/Editor-in-chief: PhD Thiago D. Pimentel (UFJF).

Recebido em 20 de Março de 2017; aceito em 20 de Junho de 2017; publicado online 24 de Julho de 2017.

Received on March 20, 2017; accepted on June 20, 2017, published online on July 24, 2017.

Artigo original de pesquisa/ Original research article. Seção revisada por pares / Double bind review section.
} 